\title{
A multi-layer bioinspired design with evolution of shish-kebab structures induced by controlled periodical shear field
}

\author{
K. J. Liu, J. Zhang* , H. Liu, X. Y. Qian, Y. Zhang, T. Wang, K. Z. Shen
}

State Key Laboratory of Polymer Materials Engineering, College of Polymer Science and Engineering, Sichuan University, Chengdu, PR China

Received 13 September 2012; accepted in revised form 9 December 2012

\begin{abstract}
The crystallization of polymers, caused by flow fields in the melt, has been the subject of extensive studies for many years. In this study, we use periodical shear to induce polypropylene to form multi-layer structure, which is usually observed in plants. Two interesting points were found: firstly, the quest of mimicking natural structures was achieved by controlled periodical shear field; secondly, the evolution from nano to shish-kebab-like cylindrite structure was obtained in the multi-layer structure, which can be clarified by nuclei competition model. This study can be used to better understand the shear-induced crystallization of polymer. Here our intention is to place this new observation on the map, leaving a fuller presentation and discussion of the work to a future publication.
\end{abstract}

Keywords: processing technologies, bioinspired, shish-kebab, multi-layer, shear-induced crystallization

\section{Introduction}

The creativity of nature is very amazing and interesting. Creatures always have the simplest but most optimized structures, e.g., reed, shell, bone and so on, which display excellent comprehensive mechanical performance, prominent adaptability and good self-healing feature. Bionics is developing rapidly as a new branch of material science, chemistry, physics and biology [1].

Reed is a large perennial grass found in wetlands throughout temperate and tropical regions of the world. The stem of reed is lightweight, high-performance structural material with exceptional strength and toughness. These two properties tend to be mutually exclusive, and attaining optimal mechanical performance is invariably a compromise often achieved through the empirical design of microstructures. Its highly sophisticated structures with complex hierarchical morphology are shown in Figure 1. Figure 1a shows that the cross section of reed stem is formed by vessels which carry nutrients and water between leaves and root. Figure $1 \mathrm{~b}$ shows that the vertical section of the reed stem which is consisted of a multi-layer structure. Figure 1c indicates that these layers are formed by vessel cells, which look like highly oriented fibers [2]. These multilayer cylindrical structures are so-called walled cylindrical shell structures [3]. Slippage between layers can absorb energy to avoid the stem breaking. The deformation of thin walled cylindrical shell structure can be restricted to deformation [4]. Can polymer products be bioinspired designed to improve their mechanical properties? The quest of mimicking natural structures in the synthesis of new structural materials has generated enormous interest but has yielded few practical advances.

\footnotetext{
${ }^{*}$ Corresponding author, e-mail: zhangjie@scu.edu.cn

(C) BME-PT
} 


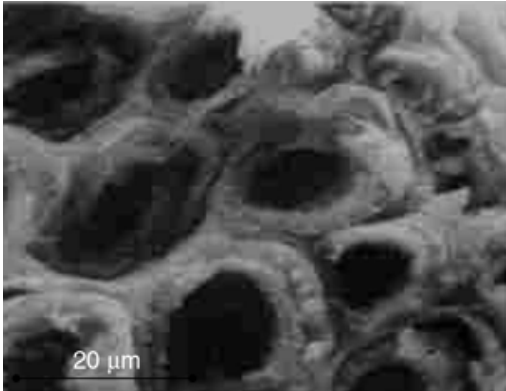

a)

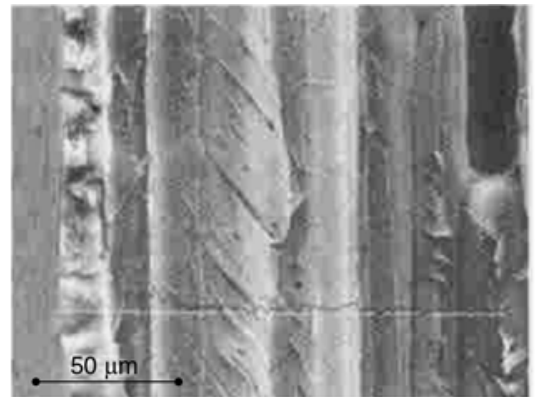

b)

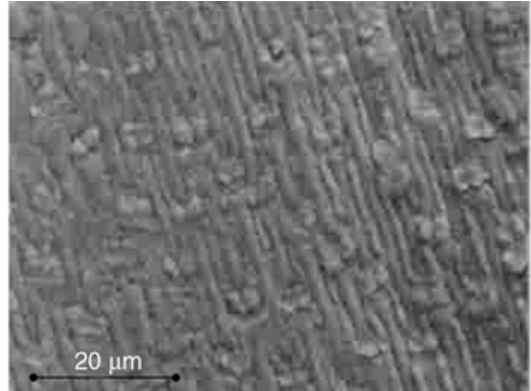

c)

Figure 1. SEM figures of reeds stem and its multi-layer structure, (a) cross section of layer from reeds stem, (b) vertical section of reeds stem, (c) surface of layer from reeds stem. (ref. [2], permitted by the Publishers)

It is well known that iPP parts prepared by injection molding always present an evolution from skin to core morphology [5, 6]. The skin [7] starts forming during the filling phase due to the rapid cooling of the hot and highly oriented melt against the cold mold wall. Transition region [8] is formed by deformed spherulites. Shear region has peculiar microstructures induced by shear flow, normally referred to as shish-kebab structures [9]. The core $[10,11]$ shows essentially $\alpha$-type spherulitic morphology. The evolution is due to the gradient of thermal and flow field. Bevis and coworkers $[12,13]$ invented shear controlled orientation injection molding (SCORIM) to provide strengthening shear field. Su et al. [14] used SCORIM to produce the so-call bioinspired bamboo-like structure with a typical hierarchic structure with high-proportion shishkebab structures in the skin and intermediate layers and abundant beta-crystals in the core layer. Another successful experiment was conducted by Zhong and coworkers $[15,16]$, who obtained injection molded polymer parts with a high and homogeneous orientation distribution upon a combination of in situ microfibrils and dynamic oscillation shear by suppressing the skin-core structure of iPP parts. Our previous work [17] also changes the morphology of injection parts by periodical vibration injection molding (PVIM).

These methods [12-17] are to strengthen shear field to induce the transition from a relatively isotropic, spherulitic morphology [18-20] to a highly oriented, shish-kebab morphology [21]. Formation of shish-kebab structure [22] can be described as follows: When polymer melts are sheared, molecules can be oriented in the flow direction. Alignment of chain segments of polymer molecules is a natural consequence of shear. At given temperature and external shear field, the degree of orientation and the extent of alignment obviously depend on the critical entanglement molecular weight, relaxation time, helical chain conformation, etc. Hence, it is possible that, under certain experimental conditions, only partial chain strands are oriented in the flow direction, while the other chain strands, especially those near the chain ends, remain unoriented or randomly oriented with respect to the flow direction. When bundles of oriented chain segments develop under shear, these bundles can initiate the formation of primary nuclei. Then, crystallization continues to produce the so-called 'shish-kebab' (cylindrite) morphology [23]. Zhang et al. [24] found the shish-kebab structures which formed at low shear rate and low temperature were different from the classical shish-kebab structures which formed at high shear rate and temperature. The greatest difference was that low temperature and shear rate can induce shish-kebab-like cylindrite structures which can be observed by polarized light microscope (PLM), while the high temperature and shear rate induced nano-scale shish-kebab structures [25], which were usually characterized by scanning electron microscope (SEM) and small angle Xray diffraction (SAXD). In our previous work [26], a region full of shish-kebab (cylindrite) structures induced by PVIM, which belong to shish-kebablike structures reported by Han and coworkers [23, 24], was also found between skin and core. These shish-kebab structures were in close resemblance to the polymorphic shish-kebab morphology in iPP melts sheared with fibers [27-29], crystallization in fibers/polymer composites [30, 31], or sample crystallization by a razor blade shear in the melt [32]. However, in our experiment there were neither fibers nor other nucleation species added.

Herein, the PVIM technique was exploited in the creation of reed-stem-like multi-layer bionic sam- 
ples. The goal was to realize the bioinspired design to significantly improve the mechanical properties of iPP samples. It was very interesting that we found the multi-layer structure composed of evolution from nano to shish-kebab-like cylindrite structure induced by periodical shear. To the best of our knowledge, this is the first report of shish-kebab structures of different scales in the same sample.

\section{Experimental section}

\subsection{Material}

IPP (grade: F401) used in the experiment was a commercial product from Lan Gang petroleum chemical, China, and its MFI is $2.3 \mathrm{~g} / 10 \mathrm{~min}$. The molecular weight of the iPP is $80 \sim 120$ thousands.

\subsection{Sample preparation}

In the PVIM, there is a vibration system in addition to injection system. The injection system provides the basic pressure while the vibration system provides oscillatory pressure. During the packing stage, a periodically changing pressure acts on the melt in the runner and mold cavity until the injection gate is frozen. A schematic representation of the melt vibration injection apparatus was shown in Figure 2. The processing parameters were shown in Table 1, which were set on the control panel. For the

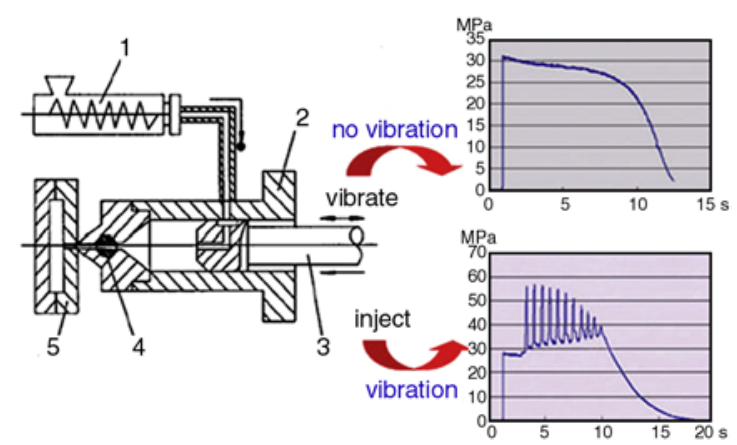

Figure 2. The schematic representation of pressure vibration injection molding and the cavity pressure profile measured from a pressure sensor. 1 - plasticizing equipment, 2 - barel, 2 - vibrating and injecting piston, 4 ; valves, 5 - injection mold

Table 1. Processing parameters

\begin{tabular}{|l|c|c|}
\hline & $\begin{array}{c}\text { Static injection } \\
\text { molding }\end{array}$ & $\begin{array}{c}\text { Vibration injection } \\
\text { molding }\end{array}$ \\
\hline Injection pressure $[\mathrm{MPa}]$ & 35 & 35 \\
\hline Packing pressure $[\mathrm{MPa}]$ & 35 & 35 \\
\hline Melt temperature $\left[{ }^{\circ} \mathrm{C}\right]$ & 200 & 200 \\
\hline Mold temperature $\left[{ }^{\circ} \mathrm{C}\right]$ & 40 & 40 \\
\hline Vibration pressure $[\mathrm{MPa}]$ & 0 & 60 \\
\hline Frequency $[\mathrm{Hz}]$ & 0 & 0.9 \\
\hline
\end{tabular}

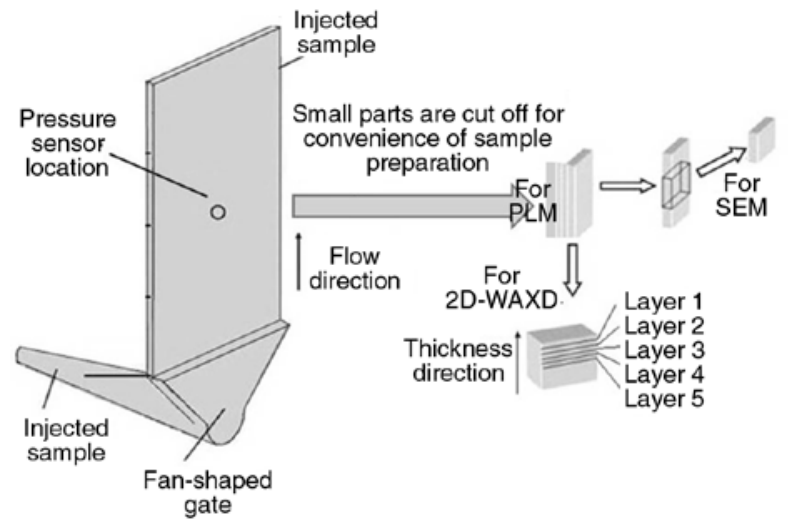

Figure 3. Specimen preparation for tests

purpose of comparison, conventional injection molding (CIM) was carried out under static packing (without vibration) by using the same processing parameters.

The samples have rectangular geometry with a dimension of $50 \mathrm{~mm} \times 80 \mathrm{~mm} \times 2 \mathrm{~mm}$. The molding gate is fan-shape with an end dimension of $50 \mathrm{~mm} \times$ $5 \mathrm{~mm}$. The thickness of gate is larger than that of cavity, so as to achieve relatively homogeneous flow. The pressure sensor was located in the central part of sample cavity. To better investigate and analyze the morphology evolution, the sample was divided into four zones along the flow direction. Besides, one half-width of each specimen was separated into five layers through the thickness direction for 2D-WAXD study, as also shown in Figue 3. Each layer is $0.2 \mathrm{~mm}$ thick. The preparation of specimen for tests is exhibited in Figure 3. The specimens for PLM observation were cut into slices with a thickness of 10 microns by means of microtome at room temperature. The microtome is MT-1 from ZheJiang Yidi Medical Apparatus and Instruments Co. Ltd., China.

\subsection{Character methods}

\subsubsection{Polarized-light microscopy (PLM)}

The specimens for PLM are $15 \mu \mathrm{m}$ thick slices prepared by microtome along MD and ND axis respectively, as shown in Figure 3. Subsequently, micrographs were taken using a DX-1 (Jiang Xi Phoenix Optical Co. China.) microscope connected with a Nikon 500D digital camera.

\subsubsection{Scanning electron microscope (SEM)}

For the SEM observation, the blocky specimen was ground and polished to the same distance from the sample surface as the slices prepared for PLM, then 
etched for a certain time in an etchant consisting of a $3 \% \mathrm{w} / \mathrm{v}$ solution of potassium permanganate dissolved in the sulphuric and dry ortho-phosphoric acids mixed solution, and then washed with $30 \%$ hydrogen peroxide and distilled water. The specimen was gold sputtered before observation.

\subsubsection{Synchrotron two-dimensional wide-angle X-ray diffraction (2D-WAXD).}

Specimens for 2D-WAXD were $1 \mathrm{~mm}$ thick slices cut from the injection sample, as shown in Figure 3. The synchrotron 2D-WAXD experiments were carried out on the U7B beam line in the National Synchrotron Radiation Laboratory (NSRL), Hefei, China. The wavelength used was $0.1409 \mathrm{~nm}$. The two-dimensional diffraction patterns were recorded every 180 s by a Mar CCD 165 X-ray detector system in transmission mode at room temperature. Azimuthally scans $\left(0-360^{\circ}\right)$ of 2D-WAXD were made for the corresponding lattice planes of the $\alpha$ form polypropylene ( $\alpha-\mathrm{PP})$. The orientation level of various planes could be calculated by the orientation parameter $\mathrm{f}$, which was calculated as shown by Equtions (1) and (2):

$f=\frac{3 \overline{\left(\cos ^{2} \phi\right)}-1}{2}$

$\overline{\left(\cos ^{2} \phi\right)}=\frac{\int_{0}^{\pi / 2} I(\phi) \sin \phi \cos ^{2} \phi \mathrm{d} \phi}{\int_{0}^{\pi / 2} I(\phi) \sin \phi \mathrm{d} \phi}$

where $\phi$ is the angle between the normal of a given crystal plane and the shear flow direction and $I$ is

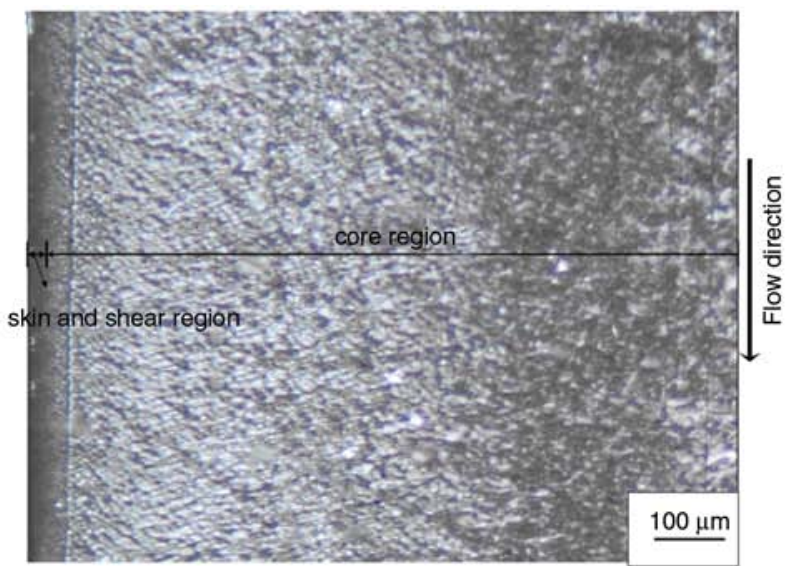

a)

Figure 4. PLM figures of (a) CIM sample and (b) PVIM sample intensity. Additionally, the relative crystallinity of $\beta$ $\left(K_{\beta}\right)$ is calculated by Equation (3):

$K_{\beta}=\frac{I_{\beta}}{I_{\alpha 1}+I_{\alpha 2}+I_{\alpha 3}+I_{\beta}}$

where $I_{\beta}$ is the intensity of the (300) reflection of the $\beta$-modification, and $I_{\alpha 1}, I_{\alpha 2}$, and $I_{\alpha 3}$ are the intensities for the (110), (040), and (130) planes of the $\alpha$-phase, respectively.

\subsubsection{Dynamic mechanical properties}

Dynamic mechanical analysis (DMA) was performed using a TA DMA Q800 analyzer from TA Instruments (USA). A three point bending clamping geometry was used. DMA tests were carried out from -100 to $150^{\circ} \mathrm{C}$ at a heating rate of $5^{\circ} \mathrm{C} / \mathrm{min}$ at $1 \mathrm{~Hz}$.

\subsubsection{Tensile testing}

Tensile testing was performed according to GBT1040-2006 (National Standards of P.R.C) on an A REGER-1030 testing machine (RTG-10) at room temperature. The tensile rate is $50 \mathrm{~mm} / \mathrm{min}$.

\section{Results and discussion}

\subsection{Hierarchical structures of CIM samples and PVIM samples by PLM}

Longitudinal sections of PVIM and CIM samples were examined using PLM. Herein, because the sample has a symmetrical structure, only half of which is shown. In Figure 4a, CIM sample can be roughly divided into skin, shear region and core region. However, because the skin and the shear region are too thin to be distinguished, they are marked together in Figure 4. That of PVIM samples

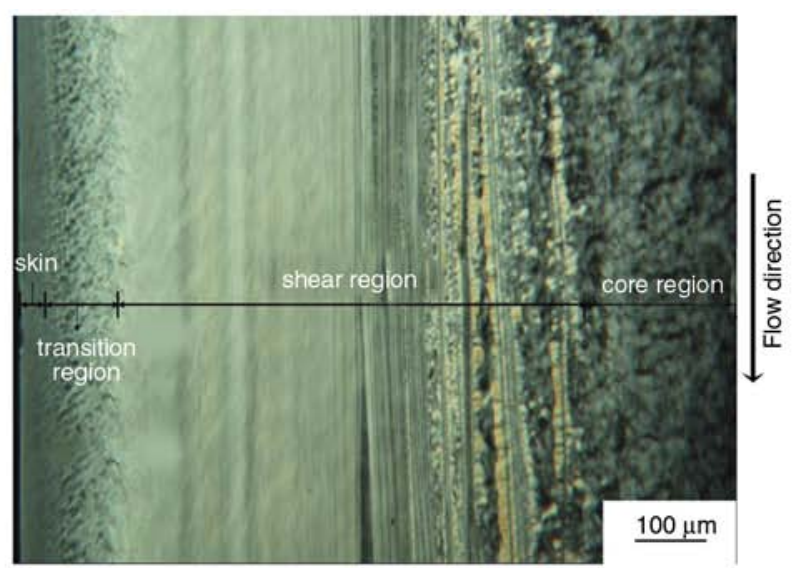

b) 
are more abundant, which can be divided into skin, transition region, shear region and core region. Skin may formed by oriented structures, which cannot be clearly observed by the PLM, as reported by other researchers $[16,17]$. Transition region is formed by transformed spherulites. Shear region consists of shear-induced crystal structures. Core region is composed of more perfect spherulites. What is notable is that obvious multi-layer structure exists in shear region, which is similar to multi-layer structure shown in Figure 1b.

In the CIM sample, the core region is the thickest, which is $972 \mu \mathrm{m}$. While, the thickness of the core region of PVIM sample is only $206 \mu \mathrm{m}$. Instead, shear region becomes the thickest in PVIM sample.

\subsection{Microstructure of multi-layer morphology by PLM and SEM}

As mentioned above, the most obvious difference between CIM sample and PVIM sample is a multilayer structure existing in the shear region of the PVIM sample as shown in Figure 5. It indicates that a so-called multi-layer morphology is formed by two obviously different layers. In the layer close to skin, there are overcrowding fiber-like [33] structures that have unclear boundaries. With the distance increasing from the skin, these fiber-like structures can be distinguished more easily. In vicinity of core, unambiguous multi-layer structure is formed by so-called shish-kebab structures.

To clarify the morphology of the two layers, their scanning electron microscopy (SEM) micrographs are presented in Figures 6 and 7. As shown in Figure 6 , the layer close to skin is also mainly constituted of shish-kebab structures. However the size of these shish-kebab structures is only a few nanome-

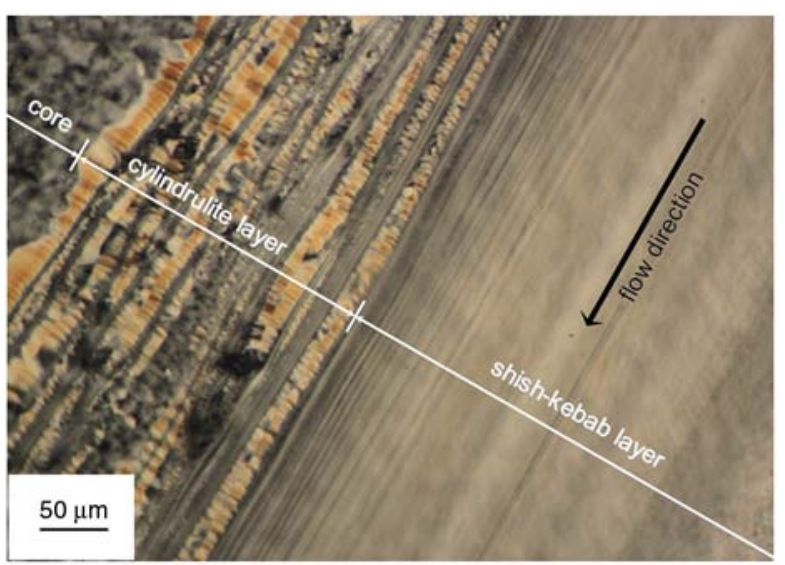

Figure 5. PLM graph of multi-layer structure of PVIM sample

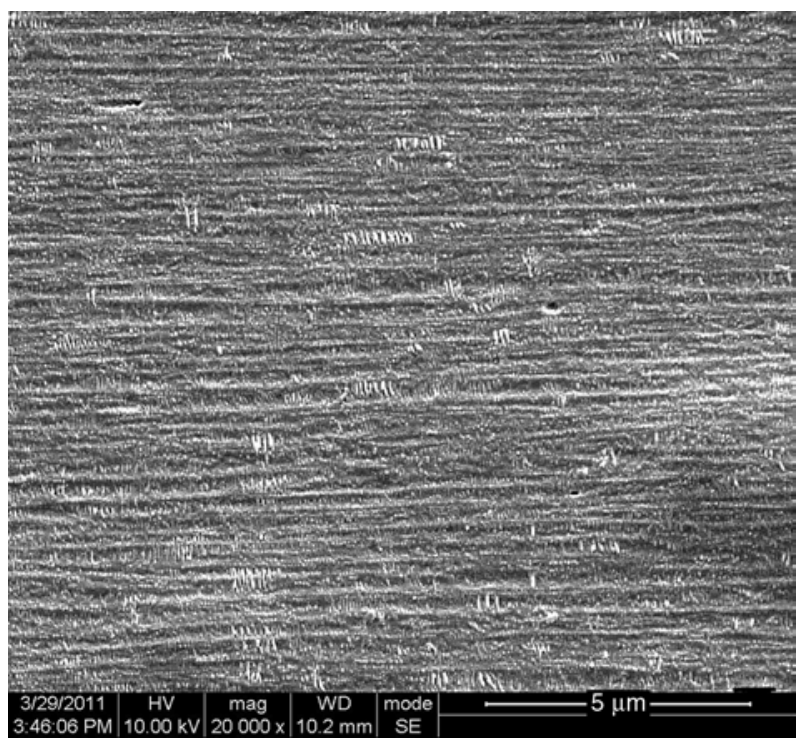

Figure 6. SEM figure of shish-kebab layer

ters. Therefore, the layer can be named as nanoscale shish-kebab layer.

The other layer contains much larger shish-kebab structures, which can be observed by PLM, is also shown by SEM in Figure 7. The layer can be named as shish-kebab-like cylindrite layer. However, the shish shown in Figure 5 is not observed, which was etched. According to the SEM figures, the shishkebab-like cylindrite structure is schematically drawn in Figure 8: in the center of the core a single fiber-like shish exists, which is induced by shear; Around the shish, the $\alpha$-to- $\beta$ growth transition or $\alpha$ $\beta$-bifurcation took place during crystal growth, which led to the formation of randomly dispersed $\beta$ nuclei; as long as the kinetic requirement for a

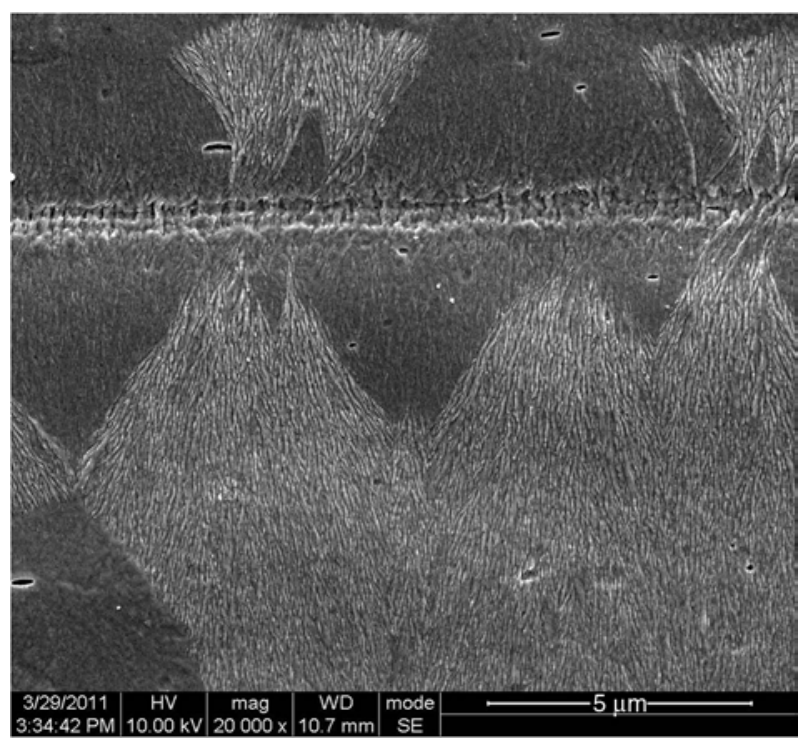

Figure 7. SEM figure of shish-kebab-like cylindrite structures 


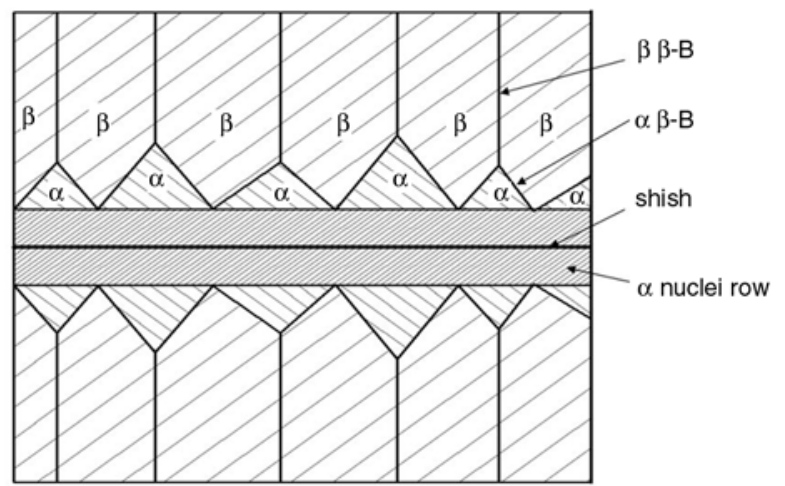

Figure 8. Schematic diagram of the cylindrite structure

higher growth rate of the $\beta$-phase $\left(G_{\beta}\right)$ than $\alpha$-phase $\left(G_{\alpha}\right)$ was achieved, these $\beta$-nuclei can induce epitaxial growth of $\beta$-phase along the $\alpha$-core [34].

The shish-kebab-like cylindrite structure with mixed $\alpha$ - and $\beta$-modification is very fascinating. The structure is expected to be able to greatly improve the mechanical performance of iPP sample. It is similar to reed-stem vessel: these kebabs are formed by $\beta$ crystal; the core is formed by rigid $\alpha$-crystals. During yielding process, acting as thin walled cylindrical shell structure in reed stem, the ductile $\beta$-crystal can deform more easily than the $\alpha$-crystal. And transformation from $\beta$-form to $\alpha$-form crystal can also absorb energy in impacting process [35].

\subsection{Orientation and crystallinity of samples by 2D-WAXD}

Figure 9 shows 2D WAXD patterns of CIM sample and PVIM sample at different distances from the skin. Except $1000 \mu \mathrm{m}$ diffraction pattern, which characterizes the structure of core, the other diffraction patterns of the PVIM sample exhibit more pronounced arcing compared with those of the CIM samples. It indicates a pronounced orientation of iPP chains within lamellae in PVIM sample.

The (040) reflection, which can be detected in all layers, is chosen to quantitatively evaluate the orientation level of CIM and PVIM samples. The orientation parameters estimated are plotted in Figure 10.

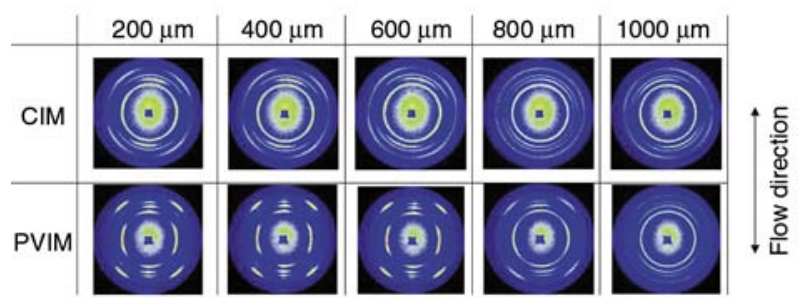

Figure 9. X-ray diffraction patterns of CIM and PVIM samples

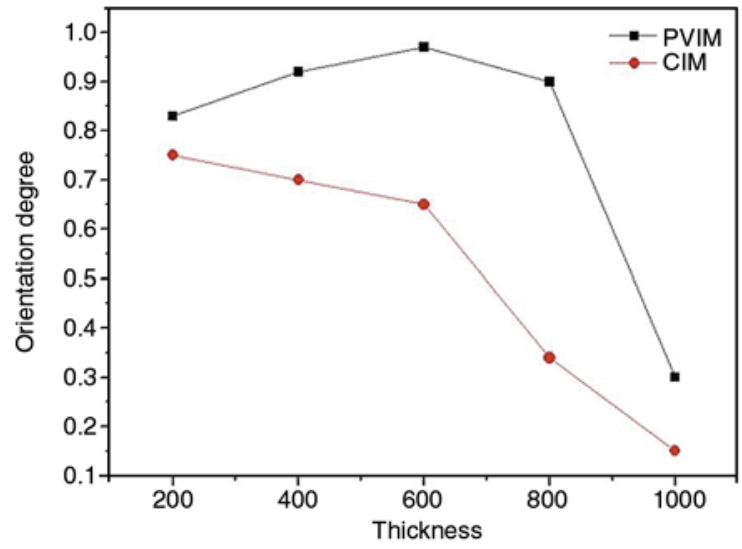

Figure 10. The evolution of orientation parameter

Orientation degree of CIM sample gradually decreases from skin $(0.75)$ to core $(0.15)$. However, orientation distribution of PVIM sample is obviously different: from 200 to $600 \mu \mathrm{m}$ in thickness direction, the orientation degree increases gradually to the highest value of 0.95 . At $800 \mu \mathrm{m}$, it is still as high as 0.9 . In the core $(1000 \mu \mathrm{m})$, it decreases to 0.3. It indicates that overall orientation of PVIM sample is higher and more narrowly distributed than CIM sample. Combined with PLM observation, an obvious conclusion can be drawn that the heterogeneity of shin-core structure of the CIM samples is suppressed to some degree in PVIM sample. In PVIM process, the formation of multi-layer morphology is a stepwise procedure. That is to say, after the solidification of one layer, on the surface of which the rest of melt is forced to flow in next oscillation period.

From these 2D-WAXD patterns in Figure 9, the $\beta$ crystal content is calculated, which is induced by shear flow. The evolution of $\beta$ relative crystal con-

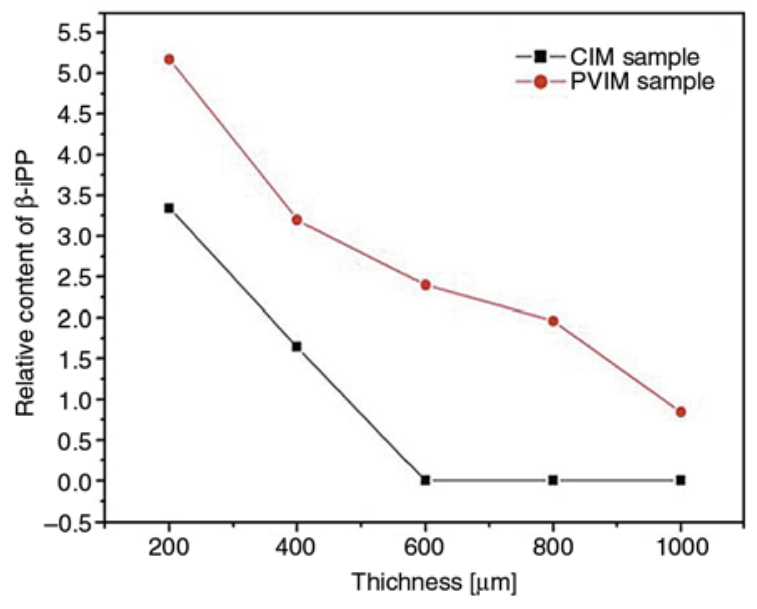

Figure 11. The evolution of $\beta$ relative crystal content 
tent is shown in Figure 11. The $\beta$ content in PVIM sample is much more than that of CIM sample. Generally, $\beta$ crystals are formed in appropriate fields, such as quenching the melt to a certain temperature range [36], directional crystallization in a thermal gradient field [37], shearing or elongation of the melt during crystallization [38], or using $\beta$ nucleating agents [39]. It should be noted that the $\beta$ crystal is also found in 800 and $1000 \mu \mathrm{m}$ layers due to optimization of shear flow by PVIM. Obviously, it is attributed to more appropriate shear and thermal field provided by PVIM than CIM.

\subsection{Formation mechanism of reed-stem-like bionic multi-layer structure}

The formation mechanism of multi-layer morphology is schematically shown in Figure 12. Before injection, iPP resin melt is in homogeneous state. Molecular chains present random coil state, as show in Figure 12(a). Under shear, molecular chain network is oriented in flow direction. This phenomenon is so-called coil-to-stretched chain transition, as show in Figure 12(b). Then, Figure 12(c) presents formation of shish from stretched chain network. These shishes act as crystal nuclei. Polymer melt crystallization is typically controlled by nucleation [40]. Lamellae epitaxially grow along shish leading to formation of plentiful shish-kebab structures, as shown in Figure 12(d), 12(e). Finally, the multilayer morphology forms, as shown in Figure 12(f). In this scenario, it's worth noting that, both nano and Shish-kebab-like cylindrite structures are formed from oriented shear-induced nucleating sites. Why the difference of dimension is so great? It is obvious that there exist three different kinds of nuclei, which can induce spherulites, shish-kebab-like and nanoscale shish-kebab, respectively. The competition between different crystal nuclei may be the reason for the difference. It is a competition for absorbing free crystalline lamellae. In shear field, the spherulite

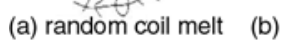

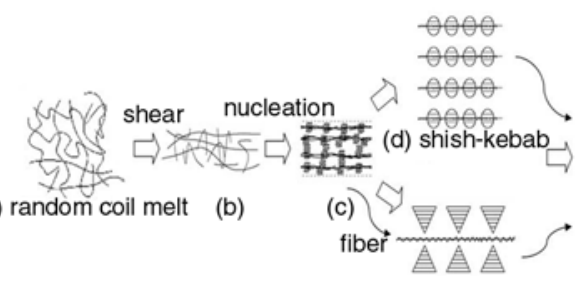

(e) shish-kebab-like cylindrite

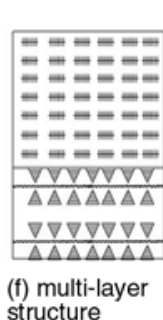

Figure 12. Schematic illustration of mechanism of the formation of multi-layer morphology growth is suppressed. Herein, we pay attention to the shear-induced structures. Although both shishkebab-like and nano-scale shish-kebab are shearinduced, their morphology is quite different. The difference is due to the distinction of density of the two layers. As shown in Figure 10, increased degree of orientation of shish-kebab layer indicates that more molecules of the layer are oriented.

As Hsiao et al. [25] proposed that kebab growth rate is diffusion-controlled. Therefore, too high density of oriented structures can hinder the molecule chains to be absorbed onto shish. Therefore, the cylindrite structures, which need about 10 20 $\mu \mathrm{m}$ space to absorb the kebab, cannot be induced in shish-kebab layer, where too little space is left to absorb enough molecules.

According to the observed morphological features, it may be taken for granted that there exists a processing parameter window, which can balance the competition of different kinds of nuclei, enables the formation of reed-stem-like multi-layer structures.

\subsection{Mechanical properties}

The tensile strength of CIM sample is $31.9 \mathrm{MPa}$. Tensile strength of PVIM sample reaches $46.7 \mathrm{MPa}$; comparing to CIM sample, it increase by $46.4 \%$. At low temperature (from -100 to $-10^{\circ} \mathrm{C}$ ), as shown in Figure 13, storage modulus of PVIM sample increases by about $25 \%$; the loss modulus of PVIM increases by $100 \mathrm{MPa}$ at low temperature. These data indicate that mechanical properties of the bioinspired sample can be improved greatly, especially high toughness of iPP parts is obtained at low temperature.

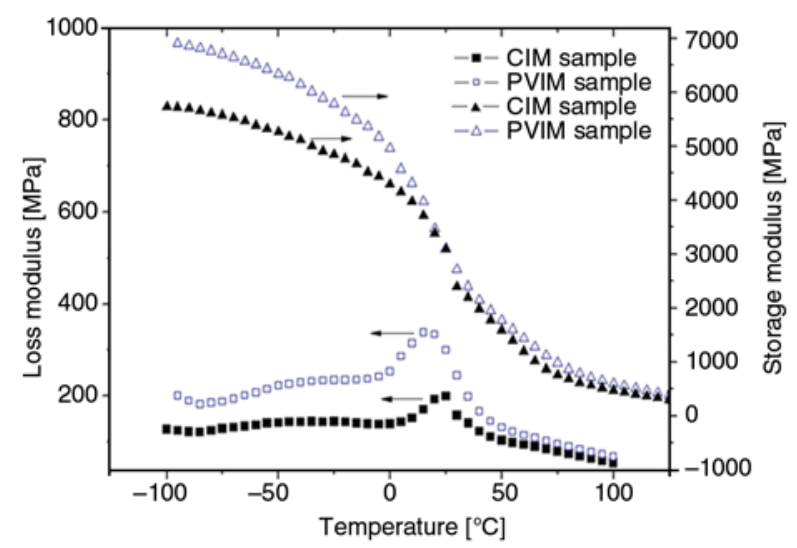

Figure 13. Mechanical properties of CIM and PVIM samples, the storage modulus and the loss modulus evolution by DMA 


\section{Conclusions}

In this study, bioinspired samples with reed-stemlike multi-layer structure were developed under periodical shear via pressure vibration injection molding (PVIM). Sample with this structure has better mechanical properties than that of CIM sample. New viewpoint is put forward about transformation from traditional skin-core structure to highly oriented and multi-layer morphology. Toughening and strengthening of iPP samples are achieved. This evolution from nano to shish-kebab-like shish kebab structures is embodied in the multi-layer structure due to gradient of shear and thermal fields. The method is feasible in industry. The result leads to new insights into the structure and formation of polymer shish-kebabs under shear flow.

\section{Acknowledgements}

We would like to express our great thanks to National Natural Science Foundation of China (50873072) and the Special Funds for State Key Laboratory for financial support. We are indebted to the National Synchrotron Radiation Laboratory (NSRL) in University of Science and Technology of China and Prof. Guoqiang Pan (NSRL) for his help in synchrotron WAXD experiment.

\section{References}

[1] Bonderer L. J., Studart A. R., Gauckler L. J.: Bioinspired design and assembly of platelet reinforced polymer films. Science, 319, 1069-1073 (2008).

DOI: $10.1126 /$ science. 1148726

[2] Yugui Q., Liansheng C.: SEM-EDAX study on reed stem (in Chinese). Transactions of China Pulp and Paper, 20, 5-7 (2005).

[3] Kim S-E., Kim C-S.: Buckling strength of the cylindrical shell and tank subjected to axially compressive loads. Thin-Walled Structures, 40, 329-353 (2002).

DOI: $10.1016 / \mathrm{s} 0263-8231(01) 00066-0$

[4] Han L., Chrysanthou A., Young K. W.: Mechanical behaviour of self-piercing riveted multi-layer joints under different specimen configurations. Materials and Design, 28, 2024-2033 (2007).

DOI: $10.1016 /$ j.matdes.2006.06.015

[5] Walker M., Smith R.: A procedure to select the best material combinations and optimally design composite sandwich cylindrical shells for minimum mass. Materials and Design, 27, 160-165 (2006).

DOI: $10.1016 / \mathrm{j}$. matdes.2004.10.003

[6] Rajesh J. J., Soulestin J., Lacrampe M. F., Krawczak P.: Effect of injection molding parameters on nanofillers dispersion in masterbatch based PP-clay nanocomposites. Express Polymer Letters, 6, 237-248 (2012).

DOI: $10.3144 /$ expresspolymlett.2012.26
[7] Viana J. C.: Structural interpretation of the strain-rate, temperature and morphology dependence of the yield stress of injection molded semicrystalline polymers. Polymer, 46, 11773-11785 (2005).

DOI: $10.1016 /$ j.polymer.2005.10.024

[8] Geng Y., Wang G., Cong Y., Bai L., Li L., Yang C.: Shear-induced nucleation and growth of long helices in supercooled isotactic polypropylene. Macromolecules, 42, 4751-4757 (2009). DOI: $10.1021 / \mathrm{ma9004567}$

[9] Ogino Y., Fukushima H., Takahashi N., Matsuba G., Nishida K., Kanaya T.: Crystallization of isotactic polypropylene under shear flow observed in a wide spatial scale. Macromolecules, 39, 7617-7625 (2006). DOI: $10.1021 / \mathrm{ma} 061254 \mathrm{t}$

[10] Somani R. H., Yang L., Hsiao B. S., Agarwal P. K., Fruitwala H. A., Tsou A. H.: Shear-induced precursor structures in isotactic polypropylene melt by in-situ rheo-SAXS and rheo-WAXD studies. Macromolecules, 35, 9096-9104 (2002).

DOI: $10.1021 / \mathrm{ma} 0207850$

[11] Somani R. H., Yang L., Hsiao B. S., Sun T., Pogodina N. V., Lustiger A.: Shear-induced molecular orientation and crystallization in isotactic polypropylene: Effects of the deformation rate and strain. Macromolecules, 38, 1244-1255 (2005). DOI: $10.1021 / \mathrm{ma} 048285 \mathrm{~d}$

[12] Mano J. F., Sousa R. A., Reis R. L., Cunha A. M., Bevis M. J.: Viscoelastic behaviour and time-temperature correspondence of HDPE with varying levels of process-induced orientation. Polymer, 42, 6187-6198 (2001).

DOI: $10.1016 / \mathrm{s} 0032-3861(01) 00090-8$

[13] Kalay G., Allan P., Bevis M. J.: $\gamma$ phase in injection moulded isotactic polypropylene. Polymer, 35, 24802482 (1994). DOI: 10.1016/0032-3861(94)90366-2

[14] Su R., Zhang Z., Gao X., Ge Y., Wang K., Fu Q.: Polypropylene injection molded part with novel macroscopic bamboo-like bionic structure. The Journal of Physical Chemistry B, 114, 9994-10001 (2010). DOI: $10.1021 /$ jp 1020802

[15] Zhong G-J., Li L., Mendes E., Byelov D., Fu Q., Li ZM.: Suppression of skin-core structure in injectionmolded polymer parts by in situ incorporation of a microfibrillar network. Macromolecules, 39, 67716775 (2006).

DOI: $10.1021 / \mathrm{ma} 0604845$

[16] Chen Y-H., Zhong G-J., Wang Y., Li Z-M., Li L.: Unusual tuning of mechanical properties of isotactic polypropylene using counteraction of shear flow and $\beta$-nucleating agent on $\beta$-form nucleation. Macromolecules, 42, 4343-4348 (2009).

DOI: $10.1021 / \mathrm{ma} 900411 \mathrm{f}$ 
[17] Zhang J., Shen K., Na S., Fu Q.: Vibration-induced change of crystal structure in isotactic polypropylene and its improved mechanical properties. Journal of Polymer Science Part B: Polymer Physics, 42, 2385 2390 (2004).

DOI: $10.1002 /$ polb.20110

[18] Kalay G., Zhong Z., Allan P., Bevis M. J.: The occurrence of the $\gamma$-phase in injection moulded polypropylene in relation to the processing conditions. Polymer, 37, 2077-2085 (1996). DOI: 10.1016/0032-3861(96)85852-6

[19] Stern C., Frick A. R., Weickert G., Michler G. H., Henning S.: Processing, morphology, and mechanical properties of liquid pool polypropylene with different molecular weights. Macromolecular Materials and Engineering, 290, 621-635 (2005).

DOI: $10.1002 /$ mame.200500081

[20] Prashantha K., Lacrampe M. F., Krawczak P.: Processing and characterization of halloysite nanotubes filled polypropylene nanocomposites based on a masterbatch route: Effect of halloysites treatment on structural and mechanical properties. Express Polymer Letters, 5, 295-307 (2011).

DOI: $10.3144 /$ expresspolymlett.2011.30

[21] Ning N., Luo F., Wang K., Zhang Q., Chen F., Du R., An C., Pan B., Fu Q.: Molecular weight dependence of hybrid shish kebab structure in injection molded bar of polyethylene/inorganic whisker composites. The Journal of Physical Chemistry B, 112, 14140-14148 (2008).

DOI: $10.1021 / \mathrm{jp} 8056515$

[22] Fang L. M., Gao P., Cao X W.: Temperature window effect and its application in extrusion of ultrahigh molecular weight polyethylene. Express Polymer Letters, 5, 674-684 (2011).

DOI: $10.3144 /$ expresspolymlett.2011.66

[23] Zhang C., Hu H., Wang X., Yao Y., Dong X., Wang D., Wang Z., Han C. C.: Formation of cylindrite structures in shear-induced crystallization of isotactic polypropylene at low shear rate. Polymer, 48, 1105-1115 (2007). DOI: $10.1016 /$ j.polymer.2006.12.025

[24] Zhang C., Hu H., Wang D., Yan S., Han C. C.: In situ optical microscope study of the shear-induced crystallization of isotactic polypropylene. Polymer, 46, 81578161 (2005).

DOI: 10.1016/j.polymer.2005.06.074

[25] Hsiao B. S., Yang L., Somani R. H., Avila-Orta C. A., Zhu L.: Unexpected shish-kebab structure in a sheared polyethylene melt. Physical Review Letters, 94, 117802/1-117802/4 (2005). DOI: 10.1103/PhysRevLett.94.117802

[26] Zhou Q., Liu F., Guo C., Fu Q., Shen K., Zhang J.: Shish-kebab-like cylindrulite structures resulted from periodical shear-induced crystallization of isotactic polypropylene. Polymer, 52, 2970-2978 (2011). DOI: $10.1016 /$ j.polymer.2011.05.002
[27] Duplay C., Monasse B., Haudin J-M., Costa J-L.: Shear-induced crystallization of polypropylene: Influence of molecular structure. Polymer International, 48, 320-326 (1999).

DOI: 10.1002/(sici)1097-0126(199904)48:4<320::aidpi164>3.0.co;2-7

[28] Varga J., Karger-Kocsis J.: Rules of supermolecular structure formation in sheared isotactic polypropylene melts. Journal of Polymer Science Part B: Polymer Physics, 34, 657-670 (1996).

DOI: $10.1002 /($ sici)1099-0488(199603)34:4<657::aidpolb6>3.0.co;2-n

[29] Wu C-M., Chen M., Karger-Kocsis J.: The role of metastability in the micromorphologic features of sheared isotactic polypropylene melts. Polymer, 40, 4195-4203 (1999). DOI: $10.1016 / \mathrm{s} 0032-3861(98) 00682-\mathrm{x}$

[30] Varga J., Karger-Kocsis J.: Direct evidence of rownucleated cylindritic crystallization in glass fiber-reinforced polypropylene composites. Polymer Bulletin, 30, 105-110 (1993). DOI: $10.1007 / \mathrm{bf00296241}$

[31] Li H., Jiang S., Wang J., Wang D., Yan S.: Optical microscopic study on the morphologies of isotactic polypropylene induced by its homogeneity fibers. Macromolecules, 36, 2802-2807 (2003).

DOI: $10.1021 / \mathrm{ma} 034062 \mathrm{w}$

[32] Bogoeva-Gaceva G., Janevski A., Mader E.: Nucleation activity of glass fibers towards iPP evaluated by DSC and polarizing light microscopy. Polymer, 42, 4409-4416 (2001).

DOI: $10.1016 / \mathrm{s} 0032-3861(00) 00659-5$

[33] Yamazaki S., Itoh M., Oka T., Kimura K.: Formation and morphology of 'shish-like' fibril crystals of aliphatic polyesters from the sheared melt. European Polymer Journal, 46, 58-68 (2010).

DOI: $10.1016 /$ j.eurpolymj.2009.09.003

[34] Hobbs J. K., Register R. A.: Imaging block copolymer crystallization in real time with the atomic force microscope. Macromolecules, 39, 703-710 (2005).

DOI: $10.1021 / \mathrm{ma} 0514020$

[35] Wang Y., Meng K., Hong S., Xie X., Zhang C., Han C. C.: Shear-induced crystallization in a blend of isotactic polypropylene and high density polyethylene. Polymer, 50, 636-644 (2009).

DOI: $10.1016 /$ j.polymer.2008.11.041

[36] Machado G., Kinast E. J., Scholten J. D., Thompson A., De Vargas T., Teixeira S. R., Samios D.: Morphological and crystalline studies of isotactic polypropylene plastically deformed and evaluated by small-angle X-ray scattering, scanning electron microscopy and Xray diffraction. European Polymer Journal, 45, 700713 (2009).

DOI: $10.1016 /$ j.eurpolymj.2008.12.015 
[37] Li J. X., Cheung W. L., Jia D.: A study on the heat of fusion of $\beta$-polypropylene. Polymer, 40, 1219-1222 (1999).

DOI: 10.1016/s0032-3861(98)00345-0

[38] Zheng G-Q., Huang L., Yang W., Yang B., Yang M-B., Li Q., Shen C-Y.: Hierarchical crystalline structure of HDPE molded by gas-assisted injection molding. Polymer, 48, 5486-5492 (2007).

DOI: $10.1016 /$ j.polymer.2007.05.047
[39] Zhao S., Xin Z., Zhang J., Han T.: Combined effect of organic phosphate sodium and nanoclay on the mechanical properties and crystallization behavior of isotactic polypropylene. Journal of Applied Polymer Science, 123, 617-626 (2012).

DOI: 10.1002 app.34527

[40] Wang M.: Hierarchical orientational relaxation inducing shish-kebab formations in polymer melt crystallization. The Journal of Physical Chemistry B, 114, 3488-3493 (2010).

DOI: $10.1021 / j p 911193 \mathrm{r}$ 